\title{
Nonperturbative quantization 'a la Heisenberg for non-Abelian gauge theories: two-equation approximation. Applications: a flux tube solution and a scalar model of glueball
}

\author{
Vladimir Dzhunushaliev ${ }^{1,2, \star}$ \\ ${ }^{1}$ IETP, Al-Farabi KazNU, Almaty, 050040, Kazakhstan \\ ${ }^{2}$ Dept. Theor. and Nucl. Phys., KazNU, Almaty, 050040, Kazakhstan
}

\begin{abstract}
The nonperturbative quantization technique à la Heisenberg is applied for the SU(3) gauge theory. The operator Yang-Mills equation and corresponding infinite set of equations for all Green's functions are considered. Gauge degrees of freedom are splitted into two groups: (1) $A_{\mu}^{a} \in S U(2) \times U(1) \subset S U(3)$; (2) coset degrees of freedom $S U(3) / S U(2) \times U(1)$. Using some assumptions about 2- and 4-point Green's functions, the infinite set of equations is truncated to two equations. The first equation is the $S U(2) \times$ $U(1)$ Yang-Mills equation, and the second equation describes a gluon condensate formed by coset fields. A flux tube solution describing longitudinal color electric fields stretched between quark and antiquark located at the \pm infinities is obtained. It is shown that the dual Meissner effect appears in this solution: the electric field is pushed out from the gluon condensate.
\end{abstract}

\section{Introduction}

One of the most important problems in modern physics is the problem of nonperturbative quantization. For example, the problem appears when explaining confinement in quantum chromodynamics. The problem is that force lines between a quark and an antiquark are confined into a flux tube and the field strength decreases exponentially with distance from quarks. This situation is fundamentally different from the situation with positive and negative charges in electrodynamics where the field strength decreases as a power law. In the 1950's, W. Heisenberg offered the procedure of nonperturbative (NP) quantization for a nonlinear spinor field [1]. Here we apply Heisenberg's idea to nonperturbative quantization of SU(3) gauge fields. This talk is based on [2].

\section{Nonperturbative quantization à la Heisenberg for non-Abelian gauge theories}

According to Heisenberg, the SU(3) Yang-Mills operator equations can be written as follows

$$
D_{v} \widehat{F}^{a \mu \nu}=0,
$$

^e-mail: v.dzhunushaliev@gmail.com 
where $\hat{F}_{\mu \nu}^{B}=\partial_{\mu} \hat{A}_{v}^{B}-\partial_{v} \hat{A}_{\mu}^{B}+g f^{B C D} \hat{A}_{\mu}^{B} \hat{A}_{v}^{D}$ is the field strength operator; $\hat{A}_{\mu}^{B}$ is the gauge potential operator; $B, C, D=1, \ldots, N$ are the SU(3) color indices; $g$ is the coupling constant; $f^{B C D}$ are the structure constants for the $\mathrm{SU}(3)$ gauge group.

In order to solve this equation, we have to write an infinite set of equations for all Green's functions

$$
\begin{aligned}
\left\langle D_{v} \widehat{F}^{A \mu v}(x)\right\rangle & =0, \\
\left\langle\hat{A}_{\alpha_{1}}^{B_{1}}\left(x_{1}\right) D_{v} \widehat{F}^{A \mu v}(x)\right\rangle & =0, \\
\left\langle\hat{A}_{\alpha_{1}}^{B_{1}}\left(x_{1}\right) \hat{A}_{\alpha_{2}}^{B_{2}}\left(x_{2}\right) D_{v} \widehat{F}^{A \mu v}(x)\right\rangle & =0, \\
\ldots & =0, \\
\left\langle\hat{A}_{\alpha_{1}}^{B_{1}}\left(x_{1}\right) \ldots \hat{A}_{\alpha_{n}}^{B_{n}}\left(x_{n}\right) D_{v} \widehat{F}^{A \mu v}(x)\right\rangle & =0 \\
\ldots & =0 .
\end{aligned}
$$

Here $\langle(\ldots)\rangle=\langle Q|(\ldots)| Q\rangle$ and $|Q\rangle$ is a quantum state of a given physical system. For brevity, we will write $\rangle$ instead of $|Q\rangle$.

The solution of the full set of equations gives us full information on the quantum state $|Q\rangle$ and field operators $\hat{A}_{\mu}^{B}$. In this sense, we can say that the solution of the full set of equations is the solution of the operator field equations. It is similar to such situation in probability theory: the knowledge of probability density is equivalent to the knowledge of all averages of a given random variable.

\subsection{Two-equation approximation}

It is not possible to solve the infinite set of equations (2)-(7). Practically we have to truncate the set of equations (2)-(7), and the truncated system will approximately describe the solution of the full system.

In order to do that, we will assume that in some physical situations all $S U(3)$ degrees of freedom can be decomposed into two groups. In the first group, the gauge fields $\hat{A}_{\mu}^{a}=\left\langle\hat{A}_{\mu}^{a}\right\rangle+i \delta \hat{A}_{\mu}^{a} \in S U(2) \times$ $U(1) \subset S U(3)$, where $\left\langle\hat{A}_{\mu}^{a}\right\rangle=A_{\mu}^{a}$ will be treated as classical fields, and $\delta \hat{A}_{\mu}^{a}$ are quantum fluctuations around the classical field $A_{\mu}^{a}$. In the second group, the gauge fields $A_{\mu}^{m} \in S U(3) / S U(2) \times U(1)$ are pure quantum ones in the sense that $\left\langle\hat{A}_{\mu}^{m}\right\rangle=0$.

We will consider physical systems where the quantum average of odd degrees of the gauge field are zero,

$$
\left\langle\left(\hat{A}_{\mu_{1}}^{m_{1}}\left(x_{1}\right) \ldots \hat{A}_{\mu_{2 k+1}}^{m_{2 k+1}}\left(x_{2 k+1}\right)\right)\right\rangle=\left\langle\left(\delta \hat{A}_{\mu_{1}}^{a_{1}}\left(x_{1}\right) \ldots \delta \hat{A}_{\mu_{2 k+1}}^{a_{2 k+1}}\left(x_{2 k+1}\right)\right)\right\rangle=0 .
$$

The decomposition of field strengths $\hat{F}_{\mu \nu}^{a}$ and $\hat{F}_{\mu \nu}^{m}$ into $(\cdots)^{a}$ and $(\cdots)^{m}$ parts can be written as follows:

$$
\begin{aligned}
& \hat{F}_{\mu \nu}^{a}=\hat{\mathcal{F}}_{\mu \nu}^{a}+g f^{a m n} \hat{A}_{\mu}^{m} \hat{A}_{v}^{n}, \\
& \hat{F}_{\mu \nu}^{m}=\partial_{[\mu} \hat{A}_{\nu]}^{m}-g f^{a m n} \hat{A}_{[\mu}^{a} \hat{A}_{\nu]}^{n}+g f^{m p q} \hat{A}_{\mu}^{p} \hat{A}_{\nu}^{q} .
\end{aligned}
$$

Here $\hat{\mathcal{F}}_{\mu \nu}^{a}=\partial_{\mu} \hat{A}_{v}^{a}-\partial_{v} \hat{A}_{\mu}^{a}+g f^{a b c} \hat{A}_{\mu}^{b} \hat{A}_{v}^{c}$ is the field strength tensor of the subgroup $S U(2) \times U(1)$; $\hat{A}_{[\mu}^{B} \hat{A}_{v]}^{C}=\hat{A}_{\mu}^{B} \hat{A}_{v}^{C}-\hat{A}_{v}^{B} \hat{A}_{\mu}^{C}$ is the antisymmetrization procedure; $a, b, c, \ldots$ are the subgroup indices $S U(2) \times U(1)$, and $m, n, p, \ldots$ are the coset indices.

Let us consider the equation (2) for $A=a$. After algebraic manipulations, we have

$$
\tilde{D}_{v} \mathcal{F}^{a \mu \nu}-\left(m^{2}\right)^{a b \mu \nu} A_{v}^{b}+\left(\mu^{2}\right)^{a b \mu v} A_{v}^{b}=j^{a \mu}
$$

where $\tilde{D}_{\mu}=\partial_{\mu}+g f^{a b c} A_{\mu}^{b}$ is the covariant derivative in the subgroup $S U(2) \times U(1)$. 2-point Green's functions for the gauge fields $\delta \hat{A}_{\mu}^{a} \in S U(2) \times U(1)$ and for the coset $A_{\mu}^{m} \in S U(N) / S U(2) \times U(1)$ are 
defined as

$$
\begin{gathered}
G^{m n \mu \nu}(y, x)=\left\langle\hat{A}^{m \mu}(y) \hat{A}^{n v}(x)\right\rangle, \\
G^{a b \mu v}(y, x)=\left\langle\delta \hat{A}^{a \mu}(y) \delta \hat{A}^{b v}(x)\right\rangle .
\end{gathered}
$$

Equation (2) for $A=m$ gives us

$$
\left\langle D_{v} \widehat{F}^{m \mu v}\right\rangle=0 \text {. }
$$

After tedious calculations, the equation (3) for $A=m, B_{1}=r, \alpha_{1}=\alpha$ gives us

$$
\begin{aligned}
& \tilde{D}_{v} \mathcal{F}^{a \mu \nu}-\left[\left(m^{2}\right)^{a b \mu \nu}-\left(\mu^{2}\right)^{a b \mu v}\right] A_{v}^{b}=j^{a \mu}, \\
& {\left[\partial_{x^{v}} \partial^{x^{\mu}} G^{r m \alpha v}(y, x)-\square_{x} G^{r m \alpha \mu}(y, x)\right]_{y=x}+} \\
& g f^{a m n}\left\{-\partial_{x^{\nu}}\left[A^{a \mu}(x) G^{r n \alpha v}(y, x)-A^{a v}(x) G^{r n \alpha \mu}(y, x)\right]_{y=x}-\right. \\
& A_{v}^{a}(x)\left[\partial^{x^{\mu}} G^{r n \alpha v}(y, x)-\partial^{x^{v}} G^{r n \alpha \mu}(y, x)\right]_{y=x}+ \\
& \left.G^{r n \alpha}{ }_{v}(x, x) \mathcal{F}^{a \mu v}(x)\right\}+g^{2} f^{a m n} f^{b n p} A_{v}^{a}(x)\left[A^{b \mu}(x) G^{r p \alpha v}(x, x)-A^{b v}(x) G^{r p \alpha \mu}(x, x)\right]+ \\
& g^{2} f^{a m n} f^{a p q} G_{v}^{r n p q \alpha}(x, x, x, x)=0,
\end{aligned}
$$

where

$$
\begin{aligned}
\left(m^{2}\right)^{a b \mu v} & =-g^{2}\left[f^{a b c} f^{c p q} G^{p q \mu v}-f^{a m n} f^{b n p}\left(\eta^{\mu v} G_{\alpha}^{m p}-G^{m p v \mu}\right)\right], \\
j^{a \mu} & =-g f^{a m n}\left\{\partial_{x^{v}} G^{m n \mu \nu}(x, x)+\left[\partial^{x^{\mu}} G_{\nu}^{m n v}(y, x)-\partial^{x^{v}} G_{v}^{m n}(y, x)\right]_{y=x}\right\}, \\
\left(\mu^{2}\right)^{a b \mu v} & =-g^{2}\left(f^{a b c} f^{c d e} G^{d e \mu v}+\eta^{\mu v} f^{a d c} f^{c b e} G_{\alpha}^{d e}+f^{a e c} f^{c d b} G^{e d v \mu}\right),
\end{aligned}
$$

and the 4-point Green's function $G_{\mu \nu \rho \sigma}^{m n p q}(x, y, z, u)=\left\langle\hat{A}_{\mu}^{m}(x) \hat{A}_{v}^{n}(y) \hat{A}_{\rho}^{p}(z) \hat{A}_{\sigma}^{q}(u)\right\rangle$.

\section{Scalar approximation for the condensate equation}

Let us consider the case when all 2-point Green's functions for coset degrees of freedom have the same order and are approximately described by one function. In this case we can assume that every 2-point Green's function can be approximately presented as

$$
G^{m n \mu v}(y, x) \approx C^{m n \mu v} D(y, x)
$$

where $C_{\mu}^{m m \mu}$ is a constant and $D(x, x)$ is an averaged dispersion of quantum field $\hat{A}_{\mu}^{m}$. We use the following $\mathfrak{2} \mathfrak{n} \mathfrak{s a t} z$ for $C^{m n \mu v}$

$$
C^{m n \mu v}=\delta^{m n} \mathcal{A}^{\mu} \mathcal{A}^{v}
$$

$\mathcal{A}^{\mu} \mathcal{A}_{\mu}$ is a constant; $\mathcal{A}^{\mu} \partial_{\mu}=\partial_{\mu} \mathcal{A}^{\mu}=0$. In order to close the equation (16), we assume that a 4-point Green's function is a bilinear combination of 2-point Green's functions:

$$
G^{m n p q \alpha \beta \mu v}(x, x, x, x) \approx C^{m n p q \alpha \beta \mu v} D(x, x)\left[M^{2}-D(x, x)\right],
$$

where $C^{m n р q \alpha \beta \mu v}$ and $M^{2}$ are constants. After that point, we obtain the following equation for the averaged dispersion of the coset quantum fields $\hat{A}_{\mu}^{m}$ :

$$
\square_{x} D(y, x)_{y=x}-\left(m_{\phi}^{2}\right)^{a b \mu v} A_{v}^{a}(x) A_{\mu}^{b}(x) D(x, x)-\lambda D(x, x)\left[M^{2}-D(x, x)\right]=0,
$$


where

$$
\begin{aligned}
\left(m_{\phi}^{2}\right)^{a b \mu v} & =g^{2} f^{a m n} f^{b n m} \frac{\mathcal{A}^{\mu} \mathcal{A}^{v}-\eta^{\mu \nu} \mathcal{A}^{\alpha} \mathcal{A}_{\alpha}}{3 \mathcal{A}^{\alpha} \mathcal{A}_{\alpha}}, \\
\lambda & =g^{2} f^{a m n} f^{a p q} \frac{C^{m n p q}{ }_{\mu \nu}{ }^{2 \nu}}{3 \mathcal{A}^{\alpha} \mathcal{A}_{\alpha}} .
\end{aligned}
$$

The solution of this equation is sought in the form

$$
D(y, x)=\phi(y) \phi(x)
$$

Substituting (26) into (23), we have the following equation for the gluon condensate $\phi$ formed by the coset quantum gauge fields $A_{\mu}^{m} \in S U(3) / S U(2) \times U(1)$

$$
\square \phi-\left(m_{\phi}^{2}\right)^{a b \mu v} A_{v}^{a} A_{\mu}^{b} \phi-\lambda \phi\left(M^{2}-\phi^{2}\right)=0 .
$$

\section{$4 \mathrm{SU}(3)$ flux tube}

In order to consider a flux tube solution describing force lines between a quark and an antiquark, let us simplify the equation (29)

$$
\begin{aligned}
\tilde{D}_{v} \mathcal{F}^{a \mu \nu}-\left[\left(m^{2}\right)^{a b \mu \nu}-\left(\mu^{2}\right)^{a b \mu \nu}\right] A_{v}^{b} & =j^{a v}, \\
\square \phi-\left(m_{\phi}^{2}\right)^{a b \mu \nu} A_{v}^{a} A_{\mu}^{b} \phi-\lambda \phi\left(M^{2}-\phi^{2}\right) & =0 .
\end{aligned}
$$

\subsection{Evaluation of $\left(\mu^{2}\right)^{a b \mu v},\left(m^{2}\right)^{a b \mu v}$, and $\left(m_{\phi}^{2}\right)^{a b \mu v}$ for the flux tube solution}

We will consider a physical system where 2-point Green's functions can approximately be expressed as follows:

$$
G^{a b \mu v}(y, x) \approx \Delta^{a b} \mathcal{B}^{\mu} \mathcal{B}^{v}
$$

where $\mathcal{B}_{\mu} \mathcal{B}^{\mu}$ is a constant and

$$
\Delta^{a b}=\left(\begin{array}{ccc}
\delta_{1} & 0 & 0 \\
0 & \delta_{2} & 0 \\
0 & 0 & \delta_{3}
\end{array}\right) .
$$

From (31) we see that our system has some color anisotropy in the $S U(2)$ subgroup: $G^{11 \mu v} \neq G^{22 \mu v} \neq$ $G^{33 \mu \nu}$. The vectors $\mathcal{A}^{\mu}$ and $\mathcal{B}^{\mu}$ are

$$
\begin{aligned}
& \mathcal{A}^{\mu}=\left(0,0,0, \frac{\mathcal{A}_{\varphi}}{\rho}\right), \\
& \mathcal{B}^{\mu}=\left(0,0, \mathcal{B}_{\rho}, \frac{\mathcal{B}_{\varphi}}{\rho}\right) .
\end{aligned}
$$

With such choice of the vectors $A_{\mu}^{a}$ and $\mathcal{B}^{\mu}$, we have

$$
\begin{aligned}
\left(\mu^{2}\right)^{1 b t v} A_{v}^{b}=g^{2}\left(\mathcal{B}_{\rho}^{2}+\mathcal{B}_{\varphi}^{2}\right)\left(\delta_{2}+\delta_{3}\right) A_{t}^{1} & =\mu_{1}^{2} A_{t}^{1}, \\
\left(\mu^{2}\right)^{2 b z v} A_{v}^{b}=-g^{2}\left(\mathcal{B}_{\rho}^{2}+\mathcal{B}_{\varphi}^{2}\right)\left(\delta_{1}+\delta_{3}\right) A_{z}^{2} & =-\mu_{2}^{2} A_{z}^{2}, \\
\left(m^{2}\right)^{1 b t v} A_{v}^{b}=\left(3 g^{2} \mathcal{A}_{\varphi}^{2}\right) A_{t}^{1} & =m^{2} A_{t}^{1}, \\
\left(m^{2}\right)^{2 b z v} A_{v}^{b}=-\left(3 g^{2} \mathcal{A}_{\varphi}^{2}\right) A_{z}^{2} & =-m^{2} A_{z}^{2}, \\
\left(m_{\phi}^{2}\right)^{a b \mu v} A_{v}^{a} A_{\mu}^{b}=\frac{g^{2}}{4}\left[\left(A_{t}^{1}\right)^{2}-\left(A_{z}^{2}\right)^{2}\right] & =m_{\phi}^{2}\left[\left(A_{t}^{1}\right)^{2}-\left(A_{z}^{2}\right)^{2}\right] .
\end{aligned}
$$


Insertion of (20) and (21) into (18) yields

$$
j^{a \mu}=0 .
$$

\subsection{Numerical solution for the flux tube}

We choose the 4-potential in the form (in the cylindrical coordinate system $t, z, \rho, \varphi$ )

$$
A_{t}^{1}(\rho)=\frac{f(\rho)}{g} ; \quad A_{z}^{2}(\rho)=\frac{v(\rho)}{g} ; \quad \phi(\rho)=\phi(\rho) .
$$

The corresponding color electric and magnetic fields are then

$$
\begin{aligned}
& E_{z}^{3}(\rho)=F_{t z}^{3}=\frac{f(\rho) v(\rho)}{g}, \\
& E_{\rho}^{1}(\rho)=F_{t \rho}^{1}=-\frac{f^{\prime}(\rho)}{g}, \\
& H_{\varphi}^{2}(\rho)=\rho \epsilon_{\varphi \rho z} F^{2 \rho z}=-\rho \frac{v^{\prime}(\rho)}{g} .
\end{aligned}
$$

Substituting (40) into the equations (28) and (29) (and after redefining $\phi=\tilde{\phi} / m, M=\tilde{M} / m$, and $\left.\lambda=m^{4} \tilde{\lambda} / g\right)$, we have

$$
\begin{aligned}
f^{\prime \prime}+\frac{f^{\prime}}{\rho} & =f\left(-v^{2}+\tilde{\phi}^{2}-\mu_{1}^{2}\right), \\
v^{\prime \prime}+\frac{v^{\prime}}{\rho} & =v\left(f^{2}+\tilde{\phi}^{2}-\mu_{2}^{2}\right), \\
\tilde{\phi}^{\prime \prime}+\frac{\tilde{\phi}^{\prime}}{\rho} & =\frac{m_{\phi}^{2}}{g^{2}} \tilde{\phi}\left[-f^{2}+v^{2}+\tilde{\lambda}\left(\tilde{\phi}^{2}-M^{2}\right)\right] .
\end{aligned}
$$

Here the prime denotes differentiation with respect to $\rho$. Equations (44)-(46) are solved as a nonlinear eigenvalue problem with the eigenvalues $\mu_{1,2}, M$ and the eigenfunctions $f, v, \tilde{\phi}$. The boundary conditions are

$$
\begin{gathered}
f(0)=0.2, f^{\prime}(0)=0 ; \\
v(0)=0.5, v^{\prime}(0)=0 ; \\
\phi(0)=1.0, \phi^{\prime}(0)=0 .
\end{gathered}
$$

The results of calculations are presented in figures 1 and 2 .

From (44)-(46) we can obtain the asymptotic behavior of the functions $f(\rho), v(x)$, and $\tilde{\phi}(\rho)$ :

$$
\begin{aligned}
& f(\rho) \approx f_{0} \frac{e^{-\rho \sqrt{M^{2}-\mu_{1}^{2}}}}{\sqrt{\rho}}, \\
& v(\rho) \approx v_{0} \frac{e^{-\rho \sqrt{M^{2}-\mu_{2}^{2}}}}{\sqrt{\rho}}, \\
& \tilde{\phi}(\rho) \approx M-\phi_{0} \frac{e^{-\rho \sqrt{2 \lambda M^{2}}}}{\sqrt{\rho}},
\end{aligned}
$$

where $f_{0}, v_{0}$, and $\phi_{0}$ are some constants. The flux of the longitudinal electric field is

$$
\Phi=\int E_{z}^{3} d s=\frac{2 \pi}{g} \int_{0}^{\infty} \rho f(\rho) v(\rho) d \rho<\infty .
$$




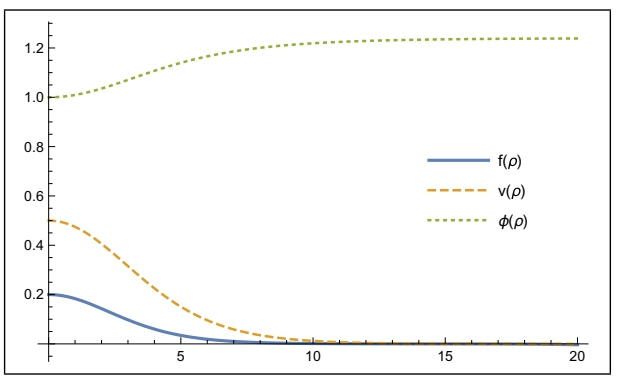

Figure 1. The functions $f(\rho), v(\rho), \tilde{\phi}(\rho)$. The solid curve is $f(\rho)$, the dashed curve is $v(\rho)$, the dotted curve is $\tilde{\phi}(\rho) . \quad \mu_{1}=1.0956253, \mu_{2}=$ $1.1494285, M=1.19505, \tilde{\lambda}=0.1$.

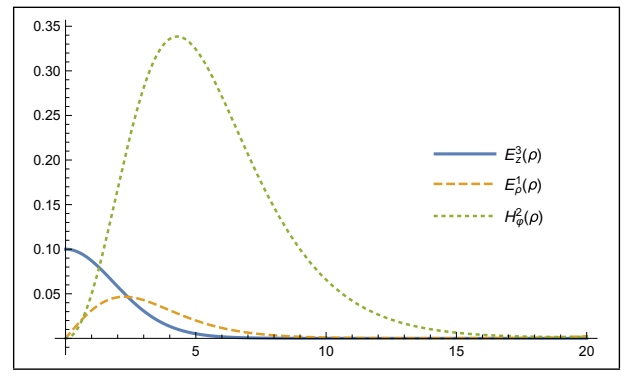

Figure 2. The chromoelectric and chromomagnetic fields $E_{z}^{3}(\rho), E_{\rho}^{1}(\rho), H_{\varphi}^{2}(\rho)$. The solid curve is $E_{z}^{3}(\rho)$, the dashed curve is $E_{\rho}^{1}(\rho)$, the dotted curve is $H_{\varphi}^{2}(\rho)$

\section{Discussion and conclusions}

- Nonperturbative quantization à la Heisenberg for QCD has been offered.

- After some simplified assumptions, the two-equation approximation has been obtained.

- The first equation is for gauge fields from a subgroup, the second one is for quantum coset fields.

- Applications: a flux tube solution, a scalar model of glueball, and the dual Meissner effect.

\section{Acknowledgements}

This work was supported by Grant $\Phi .0755$ in fundamental research in natural sciences by the MES of RK. I am very grateful to N. Kochelev and V. Folomeev for fruitful discussions and comments.

\section{References}

[1] W. Heisenberg, Introduction to the unified field theory of elementary particles., (Max - Planck Institut für Physik und Astrophysik, Interscience Publishers London, New York, Sydney, 1966), $177 \mathrm{p}$.

W. Heisenberg, Nachr. Akad. Wiss. Göttingen 8, 111 (1953).

W. Heisenberg, Zs. Naturforsch. 9a, 292 (1954).

W. Heisenberg, F. Kortel und H. Mütter, Zs. Naturforsch. 10a, 425 (1955).

W. Heisenberg, Zs. für Phys. 144, 1 (1956).

P. Askali and W. Heisenberg, Zs. Naturforsch. 12a, 177 (1957).

W. Heisenberg, Nucl. Phys. 4, 532 (1957).

W. Heisenberg, Rev. Mod. Phys. 29, 269 (1957)

[2] V. Dzhunushaliev, arXiv:1608.05662 [hep-ph] 